\title{
RESGATE DA REAÇÃO DE DEBUS-RADZISZEWSKI: ENSINO PRÁTICO DE REAÇÕES MULTICOMPONENTES NA SÍNTESE DA LOFINA
}

\author{
Victória Laysna dos Anjos Santos ${ }^{\mathrm{a}}$, Arlan de Assis Gonsalves e Cleônia Roberta Melo Araújo ${ }^{\mathrm{a}, *,(\odot)}$ \\ ${ }^{a}$ Colegiado de Ciências Farmacêuticas, Universidade Federal do Vale do São Francisco, 56304-917 Petrolina - PE, Brasil
}

Recebido em 28/04/2020; aceito em 22/06/2020; publicado na web em 07/08/2020

\begin{abstract}
RESCUE OF THE DEBUS-RADZISZEWSKI REACTION: PRACTICAL CLASS OF MULTICOMPONENT REACTIONS IN THE SYNTHESIS OF LOFIN. Multicomponent reactions are attractive for the development of bioactive substances, being a one-pot synthesis allows a quick and efficient synthesis, and allows the construction of a diversity of privileged structures. The Debus-Radziszewski reaction, a classic example of a multicomponent reaction for obtaining 2,4,5-tri-substituted imidazole, was used to compose a didactic sequence to be applied in undergraduate courses in the following disciplines: Organic Chemistry, Experimental Chemistry, Pharmaceutical Chemistry and Medicinal Chemistry. Thus, the classes were planned and applied, one theoretical and two experimental, the synthesis of lofin (2,4,5-triphenyl- $1 H$-imidazole) and the characterization of the product. The Debus-Radziszewski reaction for the preparation the lofin, proved to be suitable for practical class of multicomponent reactions, as it uses accessible reagents and equipment, it is easy to do and the students can follow the progress of the reaction visually. In addition, the didactic sequence inserts students in a contextualized and interdisciplinary way in the practical chemistry procedures, employing a classic reaction, but with current perspectives of Organic and Medicinal Chemistry, such as the synthesis of the privileged fragments of bioactive compounds.
\end{abstract}

Keywords: heterocycle; one-pot synthesis; organic chemistry class; medicinal chemistry class.

\section{INTRODUÇÃO}

As reações multicomponentes (RMCs) são definidas como processos convergentes que utilizam três ou mais reagentes de partida para obtenção de um produto final que apresente todos, ou a maior parte dos átomos de carbono presentes em seus precursores. ${ }^{1-3}$ As RMCs foram introduzidas na síntese orgânica em 1850 por Adolph Strecker, que propôs a síntese de aminoácidos a partir da hidrólise de $\alpha$-aminonitrilas, empregando uma reação entre três componentes: aldeídos, ácido cianídrico $(\mathrm{HCN})$ e amônia $\left(\mathrm{NH}_{3}\right)$. A síntese multicomponetes de compostos bioativos ganhou espaço a partir de 1882, em razão das vantagens metodológicas e de obtenção de produtos sobre a síntese linear. ${ }^{4}$

A formação do produto por meio de uma RMC acontece em etapa reacional única, denominada de reação one-pot. Nesse caso, todos os reagentes são disponibilizados no meio, porém, para que a cascata de reações seja iniciada é necessária a transformação de um dos reagentes em um intermediário mais reativo frente a outro componente disponível na mistura reacional. ${ }^{4,5}$ Sendo assim, a ordem de adição dos reagentes não interfere no processo de obtenção do produto final, de modo que são adicionados juntos e só reagirão quando o intermediário reativo for produzido. ${ }^{3}$<smiles>[R]C(=O)C([R])=O</smiles>

Glioxal: $\mathrm{R}_{1}=\mathrm{R}_{2}=\mathrm{H}$

Benzil: $\mathrm{R}_{1}=\mathrm{R}_{2}=\mathrm{Ph}$
$+\mathrm{R}_{3} \curvearrowright \mathrm{O}+$

Formaldeído: $\mathrm{R}_{3}=\mathrm{H}$ Benzaldeído: $\mathrm{R}_{3}=\mathrm{Ph}$
Considerando que as RMCs são processos one-pot, essas resultam em sínteses com tempos relativamente curtos e com elevados rendimentos, sendo estrategicamente aplicadas na Química Medicinal visando o desenvolvimento de compostos bioativos. ${ }^{6,7}$ Por se tratar, em sua maioria, de metodologias eficientes, econômicas e versáteis, a síntese multicomponente permite a obtenção de estruturas complexas, como heterociclos e policiclos heteroaromáticos com altos rendimentos reacionais, podendo assim ser utilizada na construção de quimiotecas, bibliotecas de compostos potencialmente bioativos que auxiliam na descoberta de novos compostos protótipo. ${ }^{1,2,8}$ Além disso, as RMCs são consideradas ambientalmente seguras por ocorrerem em concordância com os princípios da Química Verde, destacando-se o princípio da economia atômica, uma vez que a maioria, ou até mesmo a totalidade dos átomos de carbono presentes nos reagentes são incorporados ao arcabouço estrutural do produto final. ${ }^{7}$

A reação de Debus-Radziszewski é um exemplo de RMC, sendo resultado dos trabalhos pioneiros de Debus e Radziszewski na síntese de imidazóis. Inicialmente, em 1858, Debus propôs a síntese da glioxalina, um imidazol não substituído obtido por meio da reação entre glioxal, formaldeído e amônia (Esquema 1). Em 1882, Radziszewski e colaboradores obtiveram o composto heterocíclico 2,4,5-trifenil-1H-imidazol (a lofina) através da mesma reação, porém,<smiles>[R]c1nc([R2])c([R])[nH]1</smiles>

Amônia

Esquema 1. Reação multicomponente de Debus e Radziszewski para obtenção da glioxalina e lofina

Glioxalina: $\mathrm{R}_{1}=\mathrm{R}_{2}=\mathrm{R}_{3}=\mathrm{H}$
Lofina: $\mathrm{R}_{1}=\mathrm{R}_{2}=\mathrm{R}_{3}=\mathrm{Ph}$ 
utilizando uma $\alpha$-dicetona (benzil) como composto $\alpha$-dicarbonílico e como aldeído o benzaldeído, fato que permitiu a obtenção de um imidazol 2,4,5-trissubstituído (Esquema 1). ${ }^{9,10}$

Em decorrência dos trabalhos desses dois pesquisadores, a reação de Debus-Radziszewski é uma das metodologias mais utilizadas para obtenção de imidazóis 2,4,5-trissubstituídos. ${ }^{11}$ Ao longo dos anos, adaptações nas condições reacionais foram realizadas buscando melhores rendimentos e a síntese de derivados imidazólicos estruturalmente diversos. Dentre essas adaptações, destacam-se alterações nos substituintes dos seus reagentes convencionais: o composto $\alpha$-dicarbonilado, o aldeído e a fonte de nitrogênio. Estratégias experimentais diferentes também foram exploradas, tais como a fonte de aquecimento (convencional e micro-ondas), o emprego de suportes sólidos, solventes e catalisadores ácidos. ${ }^{12}$

A síntese de imidazóis 2,4,5-trissubstituídos por meio de RMCs possui características que atraem o interesse da indústria farmacoquímica e farmacêutica, devido à facilidade de reação, bem como a potencialidade terapêutica destes derivados que são considerados fragmentos privilegiados pela Química Medicinal, ou seja, estruturas comuns encontradas em diferentes fármacos e que apresentam relação com as diferentes atividades biológicas apresentadas. ${ }^{12,13}$

Apesar da importância das RMCs para a Síntese Orgânica e a Química Medicinal, a reação de Debus-Radziszewski ainda é pouco explorada em aulas experimentais de cursos de graduação e pós-graduação. ${ }^{7}$ Visando relembrar a reação de Debus-Radziszewski e destacar a potencialidade dos imidazóis 2,4,5-trissubstituídos, o presente artigo traz uma sequência didática que explora a síntese da lofina (Esquema 2) no ensino prático de RMCs em cursos de nível superior em Química, Farmácia, Biotecnologia, Química Medicinal, Engenharia Química e Bioquímica.

\section{PARTE EXPERIMENTAL}

A atividade prática proposta pode ser aplicada em disciplinas como Química Orgânica, Química Experimental, Química Farmacêutica, Química Medicinal e afins, com duração de duas horas cada aula. A síntese de um imidazol trissubstituído será demonstrada por meio da aplicação da reação de Debus-Radziszewski, a qual representa uma RMC clássica ao empregar três reagentes concomitantemente. Essa abordagem também permite a discussão de conteúdos secundários importantes para a química orgânica, tais como: obtenção de heterociclos potencialmente ativos, síntese sem solvente (solvent-free) e Química Verde, que podem ser previamente explorados em aulas teóricas e aplicados nesta aula prática.

A síntese proposta foi planejada visando a obtenção do 2,4,5-trifenil-1H-imidazol, um composto 2,4,5-trissubstituído potencialmente bioativo denominado de lofina, empregando-se a reação de DebusRadziszewski entre um composto $\alpha$-dicarbonilado, um aldeído aromático e uma fonte de amônia, sem a utilização de solventes (Esquema 2). ${ }^{14}$

\section{Reagentes e equipamentos}

Os reagentes empregados nessa prática foram o difenilglioxal (benzil), o benzaldeído (98\%, Neon) e o acetato de amônio (98\%, Proquimios). O benzil utilizado foi previamente sintetizado, a partir do benzaldeído, conforme descrito no material suplementar, e utilizado sem purificação prévia, uma vez que se apresentou puro após sua caracterização. $\mathrm{O}$ custo estimado para a produção do benzil foi mensurado e se encontra no material suplementar, Tabela 1S.

A reação foi realizada em tubo de reação vedado com qual foi acoplado um sistema de acondicionamento de gases preparado com material simples e de fácil acesso, uma agulha de seringa (1 $\mathrm{mm} \mathrm{x}$ $25 \mathrm{~mm}$ ) e uma bexiga, conforme demonstrado nas Figuras 1 e $2 \mathrm{~S}$. Nesse procedimento foi utilizado aquecimento convencional com banho de glicerina, agitador magnético com aquecimento $\mathrm{NOVA}^{\circledR} \mathrm{e}$ termômetro para o acompanhamento da temperatura.

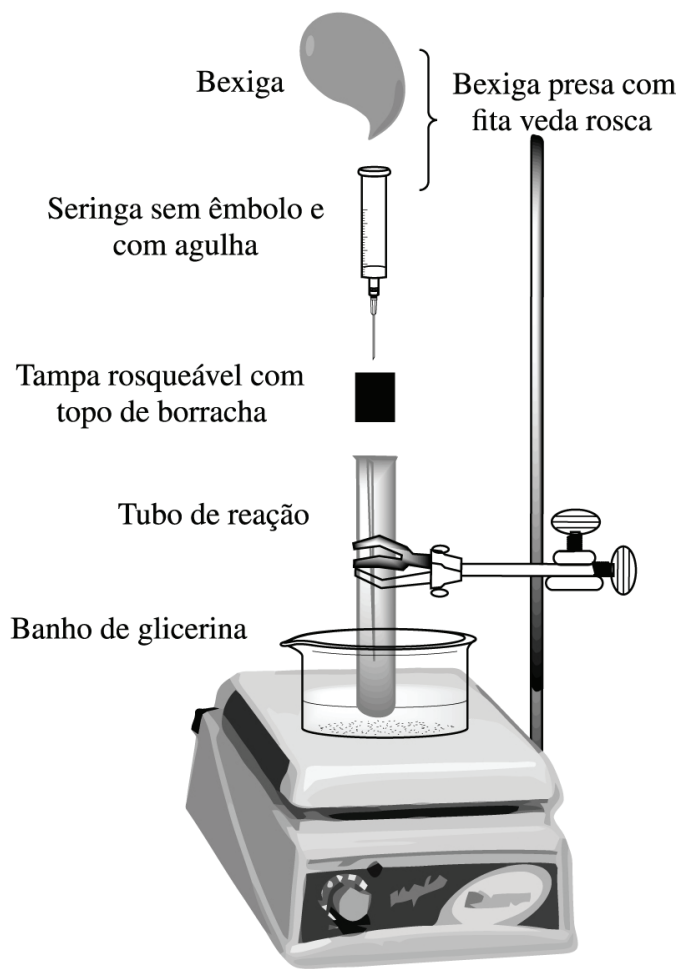

Figura 1. Representação esquemática do sistema de reação montado para a sintese da lofina

A caracterização físico-química do produto obtido foi realizada através da determinação de seu ponto de fusão (PF), utilizando fusiômetro digital Microquímica ${ }^{\circledR}$ (modelo MQAPF-302) e posterior comparação com dados descritos na literatura. Foram realizadas

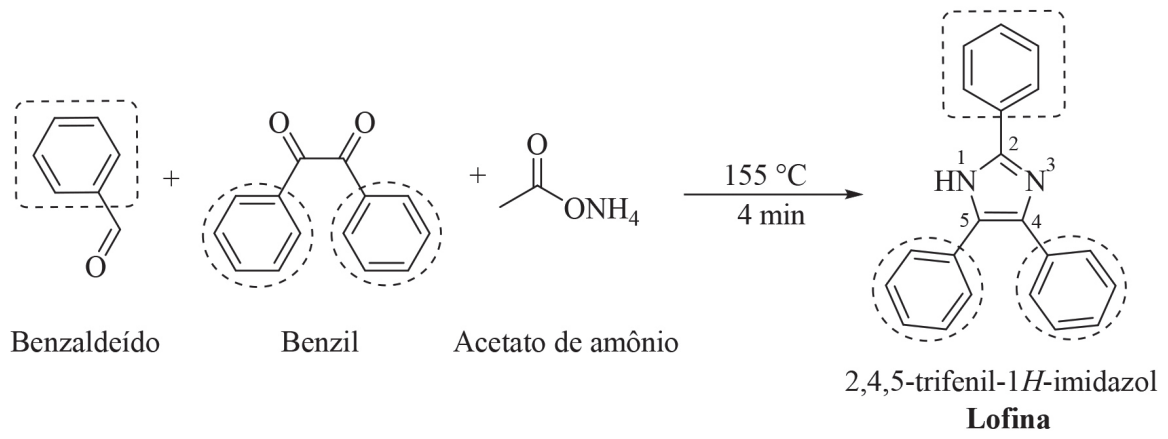

Esquema 2. Rota sintética proposta para obtenção do 2,4,5-trifenil-1H-imidazol (lofina) com destaque para os substituintes do núcleo imidazol formado nas posições 2,4 e 5 
também análises em Cromatografia em Camada Delgada Analítica (CCDA), utilizando placas de alumínio $(2 \times 4 \mathrm{~cm})$ recobertas com sílica em gel 60, com indicador fluorescente GF254 e reveladas em câmara de luz ultravioleta nos comprimentos de onda de 254 e 365 nm. Para a caracterização estrutural foram obtidos espectros de absorção na região do ultravioleta (UV-Vis) e do Infravermelho (IV) utilizando Espectrofotômetro UV-2600 e IRTracer-100 Shimadzu ${ }^{\circledR}$, respectivamente.

\section{Sequência da aula prática}

\section{Aula prática 1: síntese do 2,4,5-trifenil-1H-imidazol via RMC de Debus-Radziszewski}

Em um tubo de reação com tampa rosqueável pesou-se o benzil (1 mmol; $210 \mathrm{mg}$ ) juntamente com o acetato de amônio (15 mmol; $1,155 \mathrm{~g}$ ) e o benzaldeído ( $1 \mathrm{mmol} ; 102 \mu \mathrm{L})$. O tubo foi fechado com a tampa adaptada para evitar a perda do gás amônia formado durante a reação (Figuras 1 e 2). A mistura reacional foi submetida à agitação e aquecimento em banho de glicerina à $155^{\circ} \mathrm{C}$ por 4 minutos. $\mathrm{O}$ sistema foi resfriado até temperatura ambiente, e adicionou-se ao tubo $5 \mathrm{~mL}$ de água destilada gelada. O sólido obtido foi filtrado a vácuo e lavado com água destilada gelada.

O sólido foi purificado por meio da lavagem com clorofórmio $\left(\mathrm{CHCl}_{3}\right)$, utilizando $1 \mathrm{~mL}$ do solvente para cada $32 \mathrm{mg}$ do sólido obtido. A mistura foi aquecida em banho de glicerina à temperatura de $40{ }^{\circ} \mathrm{C}$ por cinco minutos e foi submetida a filtração simples. O sólido insolúvel no $\mathrm{CHCl}_{3}$ foi deixado secar à temperatura ambiente, obtendo-se $110 \mathrm{mg}$ do 2,4,5-trifenil-1 $H$-imidazol (lofina) na forma de um sólido amarelo pálido (Figura 3S), com 37\% de rendimento, PF: $295-298{ }^{\circ} \mathrm{C}$ (PF literatura: $280-281{ }^{\circ} \mathrm{C}$ ) e $\mathrm{F}_{\mathrm{R}}=0,43(\mathrm{AcEt} /$ Hexano $3: 7) .{ }^{15}$

\section{Aula prática 2: caracterização estrutural do 2,4,5-trifenil-1H- imidazol}

O 2,4,5-trifenil- $1 H$-imidazol (lofina) foi caracterizado através de $P F$, fator de retenção $\left(F_{R}\right)$ e espectros de absorção no UV-Vis e IV. Sugere-se que essa aula prática seja destinada à determinação do $\mathrm{PF}$, à análise do perfil cromatográfico por CCDA (sistema eluente AcEt/Hex 3:7) e a caracterização por espectrotofotometria UV-Vis.

Para obtenção dos espectros de UV-Vis foram preparadas soluções etanólicas do benzil e da lofina, na concentração de 0,017 mmol L ${ }^{-1}$, sendo etanol utilizado como "branco". As leituras foram realizadas em cubetas de quartzo, na faixa de comprimento de 200 a $500 \mathrm{~nm}$.

Para a caracterização por IV, dentro das possibilidades, recomenda-se que esses espectros sejam obtidos previamente pelo professor, de forma que tais espectros sejam mostrados e discutidos nessa aula prática. Os espectros de IV obtidos foram realizados empregando-se pastilhas de $\mathrm{KBr}$ como suporte sólido. Para o preparo da pastilha utilizou-se a proporção de $1 \mathrm{mg}$ da amostra para $100 \mathrm{mg}$ de $\mathrm{KBr}$, os quais foram macerados até obtenção de um pó fino. A mistura foi então submetida à pressão de 78,5 KN, empregando-se prensa hidráulica Shimadzu ${ }^{\circledR}$ por um período de dez minutos. Após a obtenção da pastilha de $\mathrm{KBr}$, ela foi analisada na região entre 4000 e $400 \mathrm{~cm}^{-1}$, com 45 scans e resolução de $8 \mathrm{~cm}^{-1}$.

\section{Segurança e descarte de resíduos}

Recomenda-se que a síntese do 2,4,5-trifenil-1H-imidazol (lofina) seja realizada dentro da capela de exaustão do laboratório em razão da possível liberação do gás amônia (irritante e cáustico) durante o procedimento experimental.

Os resíduos gerados nas aulas práticas, apesar de restritos, foram armazenados em frascos separados como líquidos e sólidos. Os fracos de resíduo de aulas práticas e pesquisa são recolhidos semestralmente pelo Suporte Técnico de Laboratórios da Universidade para o tratamento adequado.

\section{Estimativa dos custos para a síntese da lofina}

O custo estimado para a preparação da lofina foi calculado e encontra-se disponível no material suplementar.

\section{RESULTADOS E DISCUSSÃO}

A sequência didática foi aplicada a um grupo de seis estudantes voluntários do curso de Farmácia que já haviam cursado as disciplinas de Química Orgânica I e II, Métodos Espectrométricos, bem como Química Farmacêutica I e II. Em aula teórica, o professor abordou as RMCs e suas aplicabilidades, assim como o interesse por parte da indústria farmacêutica. Os aspectos teóricos da reação de Debus-Radziszewski também foram objetivos desse momento pedagógico.

Nessa oportunidade, o professor pôde esclarecer que estudos posteriores aos de Debus e de Radziszewski evidenciaram que a metodologia proposta por esses autores, a depender do aldeído empregado, permitia a obtenção de dois azóis isósteros: o imidazol e o oxazol. ${ }^{16}$ Esses núcleos são classificados como isósteros por apresentarem estruturas químicas diferentes, porém, com o mesmo número e/ou disposição de elétrons e o mesmo número de átomos, resultando assim na equivalência química entre esses heterociclos (Esquema 3). ${ }^{17}$ Diante disso, o docente utilizou a oportunidade para resgatar o conceito de isósteros anteriormente abordado no curso de Farmácia. Além disso, o mecanismo reacional proposto por Foerst (1964) para produzir tanto imidazóis 2,4,5-trissubstituídos quanto oxazóis 2,4,5-trissubstituídos via reação de Debus-Radziszewski foi apresentado e discutido (Esquema 3 ). ${ }^{16}$

\section{Síntese do 2,4,5-trifenil-1H-imidazol (lofina) via reação de Debus-Radziszewski}

Para a realização da síntese proposta fez-se necessário a montagem de um sistema fechado, uma vez que um de seus componentes é a amônia $\left(\mathrm{NH}_{3}\right)$, gás produzido in situ. Assim, os autores aconselham que o docente divida a turma em grupos de 2 ou 3 estudantes, e no máximo 3 grupos por aula, já que há manipulação de gás e chapa de aquecimento em alta temperatura. Recomenda-se também que o professor realize adaptações de acordo com as limitações de reagentes, equipamentos e espaço no laboratório disponíveis.

$\mathrm{Na}$ primeira aula prática de $2 \mathrm{~h}$ de duração, os estudantes foram divididos em duplas, e cada uma recebeu o roteiro experimental impresso. Os procedimentos da prática foram explicados pelo professor e as particularidades dos experimentos esclarecidos. Os estudantes montaram o sistema demonstrado nas Figuras 1 e $2 \mathrm{~S}$, em que o tubo foi fechado e a ele foi acoplada uma bexiga para acondicionamento do gás $\mathrm{NH}_{3}$ produzido durante a reação. Em seguida, foram pesados os três reagentes: benzil, benzaldeído e acetato de amônio, sendo os mesmos adicionados ao tubo de reação. $\mathrm{O}$ acetato de amônio foi utilizado como fonte de amônia para a reação, sendo utilizado em excesso (15:1) com o objetivo de favorecer a formação do gás $\mathrm{NH}_{3}$ no meio e a consequente formação do núcleo imidazol.

Diferentemente da maioria das metodologias convencionais aplicadas para a obtenção de imidazóis multissubstituídos, essa reação não requer o uso de solvente. A metodologia solvent-free resulta em uma síntese com alta eficiência, seletividade, simplicidade operacional e limpa, uma vez que corrobora com os princípios da Química Verde, como a economia de átomos, a eliminação de resíduos tóxicos, e eficiência energética e atômica. ${ }^{18}$ Além disso, há diminuição do tempo reacional, visto que após a adição dos reagentes, os choques 


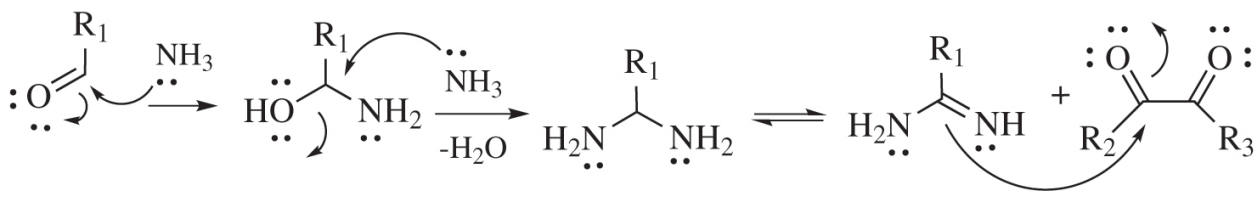<smiles>[R]C(N)=NC([R])=C([R])N</smiles>

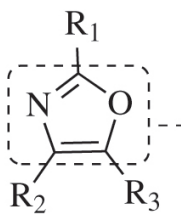

OXAZOL

\section{Ceto-enólico}<smiles>[R]C(=O)C([R2])NC(=N)NCO</smiles><smiles>[R]C(=O)C([R1])(O)NC([Y])[N]</smiles>

\section{ISÓSTEROS}<smiles>[R1]c1[nH]c(CC)c([R3])c1C=CC</smiles>

IMIDAZOL

Esquema 3. Mecanismo de formação dos azóis via reação de Debus-Radziszewski, com destaque para os isósteros sintetizados: o núcleo imidazol e oxazol 2,4,5-trissubstituído

efetivos entre as moléculas são potencializados e, consequentemente, a reação é acelerada.

A reação foi submetida à temperatura de $155^{\circ} \mathrm{C}$, em banho de glicerina e agitação por 4 min, e o progresso da reação pôde ser acompanhado visualmente pelos estudantes. $\mathrm{O}$ início do processo foi observado pelos estudantes através da fusão dos reagentes sólidos, o benzil e o acetato de amônio, uma vez que esses fundem nas temperaturas de $100{ }^{\circ} \mathrm{C}$ e $114{ }^{\circ} \mathrm{C}$, respectivamente.

Passados 2 min de reação, os estudantes notaram a formação de gás incolor $\left(\mathrm{NH}_{3}\right)$ dentro do sistema montado, através do enchimento gradual da bexiga acoplada na tampa do tubo, indicando o progresso da reação (Figura 2). A formação do gás amônia no meio requer um sistema de acomodação que evite seu escape para o ambiente, uma vez que esse reagente é a fonte de nitrogênio da reação de Debus-Radziszewski, sendo indispensável para a formação do anel imidazólico proposto. Dessa forma, é necessário que o recipiente de reação esteja devidamente tampado e acoplado com um sistema para o armazenamento do gás amônia durante a síntese. Assim, como a formação do gás no meio indica efetivamente o início da reação, logo, o esvaziamento da bexiga também pode ser utilizado como um indicador visual de que os reagentes foram completamente consumidos e que a reação deve ser encerrada (Figura 2).

A formação do produto foi evidenciada após 3 minutos, com o surgimento de um sólido amarelo pálido no meio reacional, o qual preencheu o interior do tubo. Nesse momento, o professor chamou os estudantes à reflexão: " - Como pode um sólido ter surgido se não houve redução de temperatura?" Apenas alguns discentes foram capazes de responder a esse questionamento. E logo o professor explicou que a reação teria ocorrido já que os sólidos reagentes fundem abaixo de $155^{\circ} \mathrm{C}$ e, portanto, na mistura, foi produzido um produto de ponto de fusão superior a essa temperatura. A reação foi finalizada pelos estudantes com a abertura do tubo de ensaio e a adição de água destilada ao meio reacional, sendo o sólido formado filtrado em seguida.

Usando uma pequena amostra do produto gerado na reação foi realizada uma CCDA comparativa (sistema eluente AcEt/Hex 3:7), em que os estudantes observaram o consumo total dos reagentes e a formação de dois produtos reacionais com $\mathrm{F}_{\mathrm{R}}$ distintos: 0,65 e 0,43 .

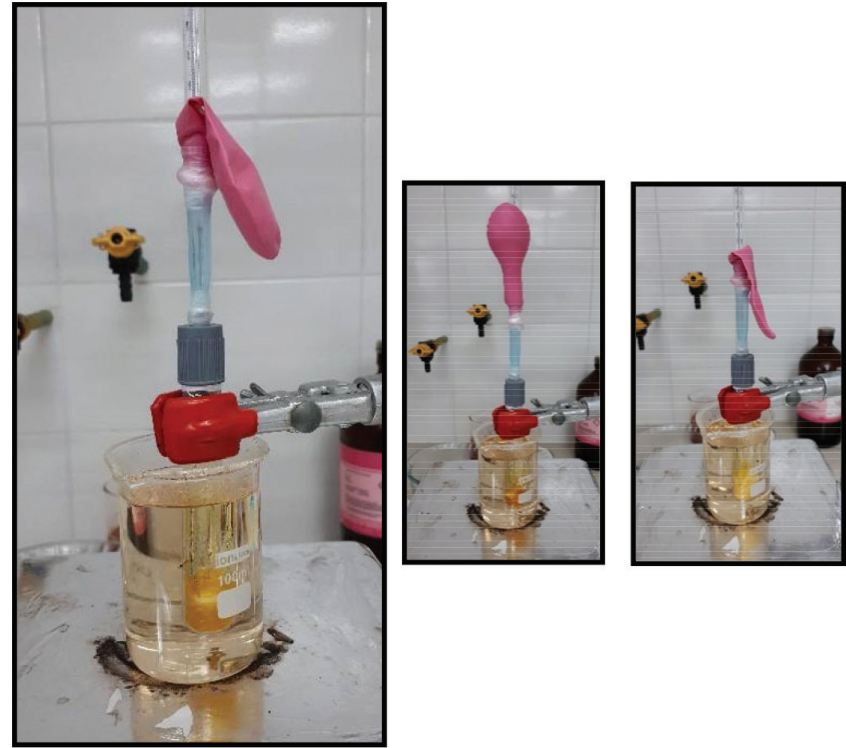

Figura 2. Progresso da reação de síntese do 2,4,5-trifenil-1H-imidazol nos tempos 0,2 e $4 \mathrm{~min}$, respectivamente

Os spots exibiram características fluorescentes semelhantes quando reveladas sob luz ultravioleta (UV) no comprimento de onda de 365 $\mathrm{nm}$ (Figura 5S). Nesse momento, o professor resgatou o mecanismo da reação de formação do núcleo imidazol, discutido anteriormente em aula teórica, e levantou a possibilidade da formação tanto do núcleo imidazol quanto do oxazol (Esquema 3).

$\mathrm{O}$ fato destes isósteros apresentarem $\mathrm{F}_{\mathrm{R}}$ distintos (Figura 5S) reforça que a substituição da porção - NH (no núcleo imidazol) por um átomo de $\mathrm{O}$ (no núcleo oxazol) implica em propriedades eletrônicas diferentes e, consequentemente, propriedades fisico-químicas distintas para cada composto. Devido à diferença de polaridade entre os produtos isósteros formados observada por CCDA (Figura 4S), foi proposta uma purificação rápida empregando-se $\mathrm{CHCl}_{3}$ como solvente. Durante a purificação foi observado que parte do produto bruto não foi solúvel no $\mathrm{CHCl}_{3}$, provavelmente o composto com menor 
$\mathrm{F}_{\mathrm{R}}$, o mais polar, e que de acordo com as características descritas na literatura, trata-se provavelmente do 2,4,5-trifenil- $1 H$-imidazol.

Apesar da simplicidade da RMC de Debus-Radziszewski, os procedimentos que os estudantes efetuaram nessa aula prática foram diversos, o que possibilita que os graduandos aprimorem suas habilidades de técnicas laboratoriais, tais como: a manipulação e pesagem dos reagentes em balança analítica, a montagem do aparato de síntese (com ênfase no uso de um reagente gasoso), filtração simples, CCDA e estratégias de isolamento e purificação.

\section{Caracterização do 2,4,5-trifenil-1H-imidazol}

Na segunda aula prática os estudantes fizeram uma CCDA (sistema eluente AcEt/Hex 3:7) do sólido obtido na aula anterior e verificaram a pureza do produto sintetizado, confirmando a eficiência da purificação (Figura 5S). Como o material estava puro e já havia secado, o sólido foi pesado e os estudantes calcularam o rendimento de obtenção do 2,4,5-trifenil-1H-imidazol após a purificação, sendo de aproximadamente $37 \%$. O rendimento foi considerado razoável, tendo em vista que a reação também proporcionou a síntese de seu isóstero e o produto bruto foi submetido a um processo de purificação.

Em seguida, o PF do produto de síntese foi determinado, sendo compreendido entre $295-298^{\circ} \mathrm{C}$. Essa faixa de PF foi comparável ao descrito na literatura para a lofina (2,4,5-trifenil- $1 H$-imidazol), indicando assim sua obtenção conforme almejado. ${ }^{15}$ Nesse momento, o professor mostrou o $\mathrm{PF}$ de seu isóstero, o composto 2,4,5-trifenil-1Hoxazol, que é de $111-113^{\circ} \mathrm{C},{ }^{19}$ bem diferente do obtido para a lofina.

Dando continuidade à caracterização do produto, os estudantes prepararam soluções etanólicas do benzaldeído, do benzil e do sólido obtido na concentração de $0,017 \mathrm{mmol} \mathrm{L}^{-1}$, e realizaram uma varredura de absorção molecular na região UV-Vis, obtendo os espectros mostrados na Figura 3. Os reagentes precursores benzaldeído e benzil mostram em seus espectros no UV-Vis bandas de absorção largas, características de transição $\pi \rightarrow \pi^{*}$ (bandas B) em 245 e $260 \mathrm{~nm}$, respectivamente, sendo resultantes do sistema de ressonância dos seus anéis aromáticos. As bandas $\mathrm{R}$ (fracas) de transição proibida $\mathrm{n} \rightarrow \pi^{*}$ das carbonilas $(\mathrm{C}=\mathrm{O})$, também esperadas para esses compostos, estão provavelmente encobertas pelas bandas B. Já o 2,4,5-trifenil-1Himidazol mostra duas bandas largas de absorção em 225 e 305 nm, sendo resultantes de transição $\pi \rightarrow \pi^{*}$ (banda $\mathrm{E}_{2}$ ) dos anéis benzênicos e de transição $n \rightarrow \pi *$ (banda $\mathrm{R}$ ) dos heteroátomos de $\mathrm{N}$ no ciclo imidazólico, respectivamente. ${ }^{20}$

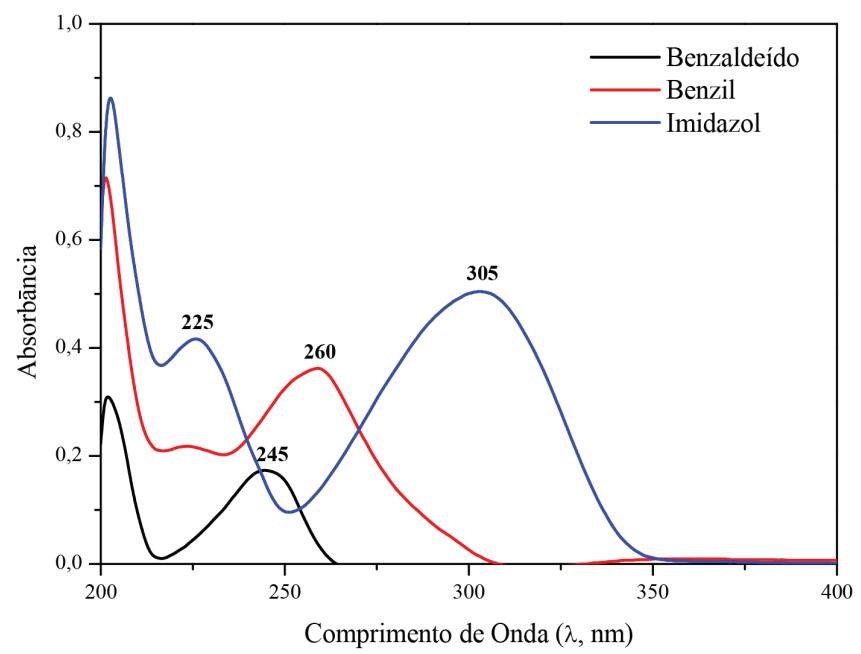

Figura 3. Espectros de absorção molecular na região UV-Vis do benzaldeído, benzil e 2,4,5-trifenil-1H-imidazol na concentração de 0,017 $\mathrm{mmol} \mathrm{L}^{-1}$
Nesse momento da aula o professor não pôde ignorar o fenômeno da quimioluminescência, já que o 2,4,5-trifenil- $1 H$-imidazol (lofina) foi o primeiro composto sintético orgânico a apresentar uma reação quimioluminescente, sendo essa descoberta em 1887 por Radiziszewski. ${ }^{21}$ Para observar esse fenômeno, os estudantes colocaram na solução etanólica já preparada para o ensaio espectrofotométrico anterior 10 gotas de uma solução de $\mathrm{NaOH}$ na concentração de $1 \%(\mathrm{~m} / \mathrm{v})$. Esse procedimento é necessário, pois, para que a quimioluminescência ocorra, a lofina necessita reagir com o oxigênio atmosférico $\left(\mathrm{O}_{2}\right)$ em meio básico e, assim, produzir um intermediário que realize a emissão (Figura 6S e Esquema 2S). Esse momento da aula prática prendeu bastante a atenção dos estudantes, despertando a curiosidade, uma vez que a visualização do fenômeno químico cria um vínculo positivo com o aprendente e auxilia no processo de aprendizagem.

Continuando com a caracterização estrutural, o professor obteve previamente os espectros vibracionais de absorção na região do IV do benzaldeído, do benzil e do produto purificado (a lofina), os quais foram apresentados e discutidos na aula prática junto ao grupo de estudantes (Figura 4, 7S, 8S e 9S). No espectro do benzaldeído foi evidente a presença dos sinais vizinhos em 2817 e $2736 \mathrm{~cm}^{-1}$, referentes ao estiramento $\mathrm{vC}-\mathrm{H}$ e característico da ressonância de Fermi observada em aldeídos. Outro sinal de ocorrência em $1700 \mathrm{~cm}^{-1}$, referente ao estiramento $\mathrm{v} \mathrm{C}=\mathrm{O}$ da carbonila, também foi observado. No espectro de IV do benzil foi observada a presença de um dubleto discreto em 1674 e $1660 \mathrm{~cm}^{-1}$, relacionado ao estiramento $v \mathrm{C}=\mathrm{O}$ e também devido à ressonância de Fermi registrada em espectros de 1,2-dicetonas com substituintes aromáticos. Além desses, foram percebidos os sinais em $3063 \mathrm{~cm}^{-1}$, do estiramento $v=\mathrm{CH}$ dos benzenos, e, em $1592 \mathrm{~cm}^{-1}$, do estiramento $\mathrm{V} \mathrm{C}=\mathrm{C}$ dos anéis aromáticos. ${ }^{22-24} \mathrm{Já}$ no espectro de IV do 2,4,5-trifenil-1 $H$-imidazol sintetizado, observou-se inicialmente a ausência das bandas em 1674, 1660 e $1592 \mathrm{~cm}^{-1}$, do reagente precursor benzil, uma vez que essas foram convertidas ao heterociclo imidazol durante a reação. A formação do núcleo imidazólico foi confirmada pela presença das seguintes bandas: i) uma banda larga de absorção em $3456 \mathrm{~cm}^{-1}$, característica do estiramento ৩N-H aromático; ii) diversas bandas na região em torno de $3000 \mathrm{~cm}^{-1}$, com máximo em $3036 \mathrm{~cm}^{-1}$, referentes ao estiramento $v=\mathrm{CH}$ do anel imidazol; iii) uma banda em $1602 \mathrm{~cm}^{-1}$, referente aos estiramentos v $=\mathrm{N}$ e $v \mathrm{C}=\mathrm{C}$; e iv) uma banda em $1127 \mathrm{~cm}^{-1}$, correspondente ao estiramento do tipo $\mathrm{vC}-\mathrm{N}$, Figura 4, 7S, 8S e 9S. .,22,23 $^{-1}$

Ao realizar a caracterização físico-química e estrutural, os estudantes confirmaram a obtenção da lofina, com rendimento considerado razoável e tempo reacional extremamente curto. Atividades com esse caráter prático tornam possível a aprendizagem cognitiva no que se refere ao conhecer e compreender, assim como a aprendizagem psicomotora durante as etapas da manipulação dos reagentes, equipamentos e materiais. Além disso, a atividade prática também pode levar ao conhecimento afetivo, no qual o estudante é valorizado e capaz de se orgulhar dos resultados exitosos obtidos ao final de seus experimentos.

\section{CONCLUSÕES}

A reação de Debus-Radziszewski para a preparação da lofina, o 2,4,5-trifenil- $H$-imidazol, mostrou-se pertinente para o ensino das RMCs. Dentre as vantagens que podem ser destacadas estão a facilidade na obtenção dos reagentes, o uso de equipamentos acessíveis, a fácil execução e o acompanhamento visual do progresso da reação. Além disso, a presente prática insere os discentes de forma contextualizada e interdisciplinar em vários procedimentos no laboratório de química, tudo isso empregando uma reação clássica, porém, com perspectivas atuais da Química Orgânica e Medicinal, como a inserção 


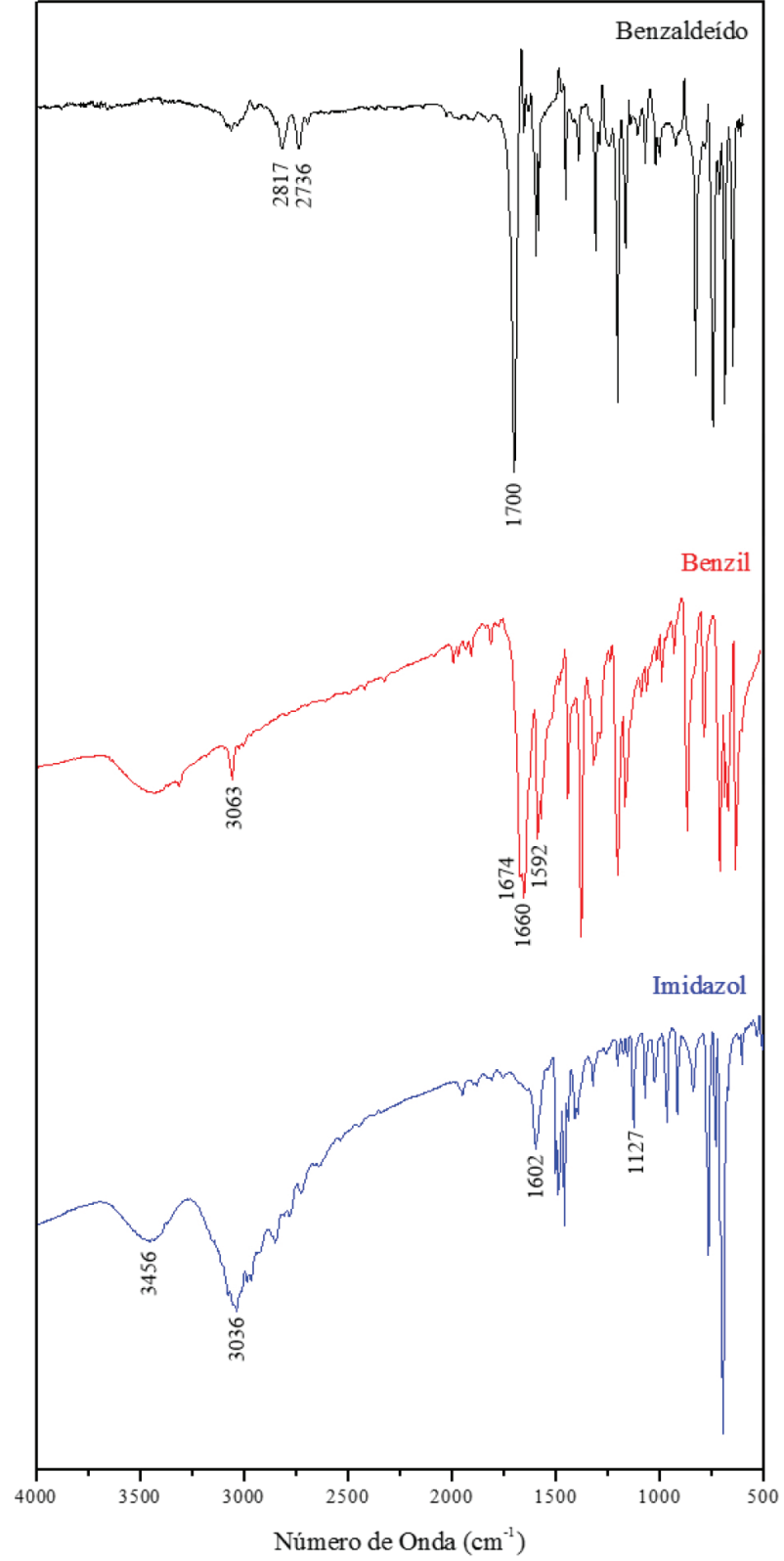

Figura 4. Espectro de absorção no IV do benzaldeído, benzil e 2,4,5-trifenil-1H-imidazol

de heterociclos considerados fragmentos privilegiados em compostos bioativos e a aplicabilidade da Química Verde.

\section{MATERIAL SUPLEMENTAR}

A metodologia de síntese do benzil a partir do benzaldeído, assim como as imagens do sistema produzido para realização da reação, das CCDAs e os espectros vibracionais de absorção no IV, estão disponíveis em http://quimicanova.sbq.org.br, na forma de arquivo PDF, com acesso livre.

\section{AGRADECIMENTOS}

Os autores agradecem à Universidade Federal do Vale do São Francisco - UNIVASF - pelo apoio e infraestrutura para realização dos experimentos

\section{REFERÊNCIAS}

1. Batalha, P. N.; Rev. Virtual Quim. 2012, 4, 13.

2. Graaff, C.; Ruijter, E.; Orru, R. V. A.; Chem. Soc. Rev. 2012, 41, 3969

3. Biggs-Houck, J. E.; Younai, A.; Shaw, J. T.; Curr. Opin. Chem. Biol. 2010, 14, 371 .

4. Rogerio, K. R.; Vitório, F.; Kümmerle, A. E.; Graebin, C. S.; Rev. Virtual Quim. 2016, 8, 1934.

5. Tejero, T. N.; Kümmerle, A. E.; Bauerfeldt, G. F.; Rev. Virtual Quim. 2019, 11, 1203.

6. Sonar, J.; Pardeshi1, S.; Dokhe, S.; Pawar, R.; Kharat, K.; Zine, A.; Matsagar, B.; Wu, K.; Thore, S.; SN Appl. Sci. 2019, 1, 1045.

7. Marques, M. V.; Bisol, T. B.; Sá, M. M.; Quim. Nova 2012, 35, 1696.

8. Galli, U.; Hysenlika, R.; Meneghetti, F.; Grosso, E. D.; Pelliccia, S.; Novellino, E.; Giustiniano, M.; Tron, G. C.; Molecules 2019, 24, 1959.

9. Kerru, N.; Bhaskaruni, S. V. H. S.; Gummidi, L.; Maddila, S. N.; Maddila, S.; Jonnalagadda, S.; B. Synth. Commun. 2019, 49, 2437.

10. Wang, Q-D.; Yang, J-M.; Zhou, B.; Fang, D.; Ren, J.; Zeng, B-B.; ChemistrySelect 2017, 2, 4807.

11. Bansal, R.; Soni, P. K.; Halve, A. K.; J. Heterocycl. Chem. 2018, 55, 1308.

12. Saxer, S.; Marestin, C.; Merciera, R.; Dupuy, J.; Polym. Chem. 2018, 9 , 1927.

13. Montanari, C. A. Em Química medicinal: métodos e fundamentos em planejamento de fármacos; Honório, K. M., Andricopulo, A. D., eds.; Edusp: São Paulo, 2011.

14. Pradhan, K.; Tiwary, B. K.; Hossain, M; Chakraborty, R.; Nanda, A. K.; RSC Adv. 2016, 6, 10743.

15. Wang, X. C.; Gong, H. P.; Quan, Z. J.; Li, L.; Ye, H. L.; Chin. Chem. Lett. 2009, 20, 44

16. Foerst, W. Em Newer Methods of Preparative Organic Chemistry; Bredereck, H., Gompper, B., Schuh, H. G. V., Theilig, G., eds.; Academic Press: New York, London, 1964.

17. Wermuth, C. G. Em The Practice of Medicinal Chemistry; Ciapetti, P., Giethlen, B., eds.; Academic Press: Illkirch, 2011, cap. 15.

18. Elinson, M. N.; Vereshchagin, A. N.; Ryzhkov, F. V.; Anisina, Y. E.; ARKIVOC 2018, 276.

19. Marques, M. V.; Ruthner, M. M.; Fontoura, L. A. M.; Russowsky, D.; J. Braz. Chem. Soc. 2012, 23, 171.

20. Silverstein, R. M.; Bassler, G. C.; Morrill, T. C.; Identificação espectrométrica de compostos orgânicos, $3^{\mathrm{a}}$ ed., Guanabara Dois: Rio de Janeiro, 1979.

21. Alves, J.; Boaro, A.; Silva, J. S.; Ferreira, T. L.; Keslarek, V. B.; Cabral, C. A.; Orfão Jr., R. B.; Ciscato. L. F. M. L.; Bartoloni, F. H.; Photochem. Photobiol. Sci. 2015, 14, 320 .

22. Barbosa, L. C. A.; Espectroscopia no Infravermelho, $1^{\text {a }}$ ed., $3^{\text {a }}$ impressão, Editora UFV: Viçosa, 2013.

23. Pavia, D. L.; Lampan, G. M.; Kriz, G. S.; Vyvyan, J. R.; Introdução à Espectroscopia, $4^{\mathrm{a}}$ ed., Cengage Learning: São Paulo, 2010.

24. Chen, M.; Zhao, Q.; She, D-B.; Yang, M-Y.; Hui, H-H.; Huang, G-S.; J. Chem. Sci. (Amritsar, India) 2008, 119, 347. 